\title{
Rapid Prediction of Possible Inhibitors for SARS-CoV-2 Main Protease using Docking and FPL Simulations
}

\author{
Minh Quan Pham ${ }^{a, b}$, Khanh B. Vu, ${ }^{c, d}$ T. Ngoc Han Pham, ${ }^{e}$ Le Thi Thuy Huong, ${ }^{\text {a,b }}$ Linh Hoang \\ Tran, ${ }^{\mathrm{d}, \mathrm{f}}$ Nguyen Thanh Tung, ${ }^{\mathrm{a}, \mathrm{g}}$ Van V. Vu, ${ }^{\mathrm{h}}$ Trung Hai Nguyen, ${ }^{\mathrm{ij}}$ and Son Tung Ngo ${ }^{\mathrm{i}, j *}$ \\ a Graduate University of Science and Technology, Vietnam Academy of Science and Technology, Hanoi, \\ Vietnam \\ b. Institute of Natural Products Chemistry, Vietnam Academy of Science and Technology, Hanoi, Vietnam \\ c. School of Biotechnology, International University, Ho Chi Minh City, Vietnam \\ d. Vietnam National University, Ho Chi Minh City, Vietnam \\ e. Faculty of Pharmacy, Ton Duc Thang University, Ho Chi Minh City, Vietnam \\ f. Faculty of Civil Energeering, Ho Chi Minh University of Technology (HCMUT), Ho Chi Minh, Vietnam \\ g. Institute of Materials Science, Vietnam Academy of Science and Technology, Hanoi, Vietnam \\ h. NTT Hi-Tech Institute, Nguyen Tat Thanh University, Ho Chi Minh City, Vietnam \\ i. Laboratory of Theoretical and Computational Biophysics, Ton Duc Thang University, Ho Chi Minh City, \\ Vietnam. \\ j. Faculty of Applied Sciences, Ton Duc Thang University, Ho Chi Minh City, Vietnam. \\ *Email: ngosontung@tdtu.edu.vn
}

\begin{abstract}
Appearance for the first time from Wuhan, China, the SARS-CoV-2 rapidly outbreaks worldwide and causes a serious global health issue. The effective treatment for SARS-CoV-2 is still unavailable. Therefore, in this work, we have tried to rapidly predict a list of potential inhibitors for SARS-CoV-2 main protease (Mpro) using a combination of molecular docking and fast pulling of ligand (FPL) simulations. The approaches were initially validated over a set of eleven available inhibitors. Both Autodock Vina and FPL calculations adopted good consistent results with the respective experiment with correlation coefficients of $R_{\text {Dock }}=0.72 \pm 0.14$ and $R_{\mathrm{W}}=-0.76 \pm 0.10$, respectively. The combined approaches were then utilized to predict possible inhibitors, which were selected from a ZINC15 sub-database, for SARS-CoV-2 Mpro. Twenty compounds were suggested to be able to bind well to SARS-CoV-2 Mpro. Among them, five top-leads are periandrin $V$, penimocycline, cis-p-Coumaroylcorosolic acid, glycyrrhizin, and uralsaponin B. The obtained results probably lead to enhance COVID-19 therapy.
\end{abstract}

\section{Introduction}

The novel coronavirus, named SARS-CoV-2 or 2019-nCoV, causes COVID-19 disease which is an ongoing global pandemic. First cases of COVID-19 infection were reported in Wuhan, Hubei, China in December 2019. ${ }^{1-3}$ The virus was found to be able to transmit from human to human. ${ }^{4}$ Especially, It has been suggested that SARS-CoV-2 can transmit through airborne/aerosol since the virus was found to remain viable and infectious in such environment for more than three hours. ${ }^{5}$ The novel coronavirus causes severe acute respiratory syndromes which have resulted in hundreds of thousands of deaths worldwide. ${ }^{6,7}$ Moreover, the intermediate host is still undetected, although the original reservoir is indicated as the bat. ${ }^{8}$ Understanding the spread of the virus thus becomes more difficult. The current global health crisis caused by COVID-19 has called for urgent research and development of an efficient antiviral drug.

SARS-CoV-2 and SARS-CoV share about $82 \%$ similarity in their RNA genomes. The genomes of coronaviruses ranges from 26 to $32 \mathrm{~kb}$ in length. The viruses thus have the largest sequence among RNA virus. ${ }^{9}$, ${ }^{10}$ There are more than 20 different proteins encoded by the genomes of the SARS-CoV-2. In particular, the SARS-CoV-2 Mpro is known as one of the most critical viral proteins. It should be noted that the SARS-CoV-2 Mpro adopts more than $96 \%$ similarity to the SARS-CoV Mpro. During the viral translation, the SARS-CoV-2 Mpro cleaves eleven polyproteins to polypeptides, which are necessary for the transcription and replication of the virus. ${ }^{10}$ The SARS-CoV-2 Mpro is selected as one of the most potent drug targets for inhibiting the viral proliferations. ${ }^{11,12}$ Thefore, numerous studies have been conducted using experimental and computational approaches in order to search for potential small-molecule inhibitors that can effectively block the activity of this protease. ${ }^{11-18}$

It should be noted that computer-aided drug design (CADD) can significantly reduce the time and cost for developing a therapy. ${ }^{19}, 20$ In the CADD approach, the ligand-binding free energy $\Delta G$ can be calculated through MD simulations. This metric is linked with the experiment through formula $\Delta G_{\text {bind }}=R \operatorname{Rn}\left(k_{\mathrm{i}}\right)$, where $k_{\mathrm{i}}$ is inhibition constant, $T$ is absolute temperature, and $R$ is gas constant. In some cases, IC50 is assumed to be equal to $k_{\mathrm{i}}$ in order to estimate the experimental binding free energy $\Delta G_{\mathrm{EXP}}{ }^{15,21,22}$ Because the metric reveals the binding mechanism between biomolecules, ${ }^{19}$ accurate and precise investigation of the ligand-binding free energy is tremendously critical for searching potential inhibitors. ${ }^{23}$ In this work, the potential candidates from ZINC15 in man compounds, ${ }^{24}$ which can bind to the SARS-CoV-2 Mpro, were screened using combined approaches of molecular docking and FPL simulations. It should be noted that this combined computational scheme was previously validated by testing on eleven available inhibitors whose computed binding free energies were in good agreement with respect to experiments. ${ }^{11-14}$ Our present study suggested that twenty compounds were able to bind with high affinity to SARS-CoV-2 Mpro. These compounds can become promising leads for developing drugs against the COVID-19 disease.

\section{Materials and Methods}

Structure of Ligands and SARS-CoV-2 Mpro 
The crystal structure of monomeric SARS-COV-2 Mpro was obtained from the Protein Data Bank with the identify of $6 \mathrm{Y} 2 \mathrm{~F} .{ }^{11} \mathrm{It}$ should be noted that computational investigations of promising inhibitors for SARS-CoV-2 Mpro are possible for the monomeric form, 25,26 because the SARS-CoV-2 Mpro dimer interface does not contain the substrate-binding cleft. ${ }^{11,12}$ Ligand structures were taken from the ZINC15 in man only and the PubChem database. ${ }^{24,27}$

\section{Molecular Docking Simulations}

The ligands were docked to the SARS-CoV-2 Mpro by using the Autodock Vina version 1.1 package. ${ }^{28}$ The parameter of the docking approach was preferred according to the previous study, ${ }^{15,29}$ in which the parameter of exhaustiveness was set to the default value of 8 . The best docking conformations were chosen as having the largest binding affinity. The grid center was designated as the center of mass of compound $\alpha$-ketoamide $\mathbf{1 3 b}$. $^{11}$ The docking grid was chosen as $2.6 \times 2.6 \times 2.6 \mathrm{~nm}$ according to the recent work. ${ }^{15}$

\section{Fast Pulling of Ligand (FPL) Simulations}

GROMACS version $5.1 .5^{30}$ was utilized to simulate unbinding process of a ligand pulled out of the binding site of the SARS-CoV-2 Mpro. The protein and ions were parameterized via the Amber99SBILDN force field. ${ }^{31}$ The TIP3P model was utilized for representing water molecules. ${ }^{32}$ The general Amber force field (GAFF) ${ }^{33}$ was used to represent the ligand via AmberTools $18 .{ }^{34}$ The ACPYPE ${ }^{35}$ protocol was used to transform AMBER to GROMACS formats. In particular, the ligand atomic charges were fitted using the restrained electrostatic potential (RESP) method ${ }^{36}$ which is based on DFT calculations by with the B3LYP functional and $6-31 G(d, p)$ basis set. It should be noted that the combination of the force fields was preferred since it is one of the most solution for free energy assessment. ${ }^{37,38}$

The complex SARS-CoV-2 + ligand was initially introduced into a rectangular PBC (periodic boundary conditions) box with a size of $9.83 \times 5.92 \times 8.70 \mathrm{~nm}$ (Figure 1), similarly to the recent work. ${ }^{15}$ The complex system thus consists of more than 50000 atoms including the SARS-CoV-2 Mpro, inhibitor, water molecules, and counterbalanced ions $\mathrm{Na}^{+}$. The solvated SARS-CoV-2 Mpro + ligand system was firstly minimized via the steepest descent approach. The $0.1 \mathrm{~ns}$ of NVT and $2.0 \mathrm{~ns}$ of NPT imitations were then followed to relax the complexed system, in which the SARS-CoV-2 Mpro $C_{\alpha}$ atoms were positionally restrained using a slight harmonic force. Finally, the ligand was forced to move out of the SARS-CoV-2 Mpro binding pocket by applying an external harmonic force with a pulling speed of $k=0.005 \mathrm{~nm} \mathrm{ps}^{-1}$ and a spring constant of $v=600 \mathrm{~kJ} \mathrm{~mol}^{-1} \mathrm{~nm}^{-2}$. The pulling speed and spring constant were chosen to be the same as in the previous works. ${ }^{15,} 39,40$ During steered-MD simulations, the ligand displacement and the applied pulling force were recorded every 0.1 ps that would be used to estimate the ligand binding affinity. ${ }^{39}$ Totally, the FPL calculations were independently performed 8 times to guarantee the sufficient sampling.

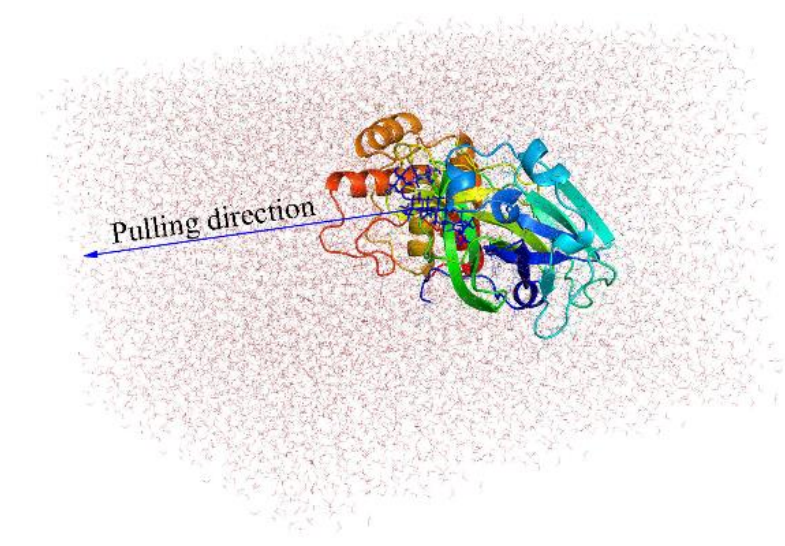

Figure 1. Initial conformation of FPL simulations of the SARS-CoV-2 Mpro + periandrin $\checkmark$.

\section{Analyzed Tools}

The ligand protonation state was predicted by using the Chemicalize tools (www.chemicalize.com), a website application of the ChemAxon. The error of computations was computed through 1000 rounds of the bootstrapping method. ${ }^{41}$ The protein-ligand interaction illustration was prepared via LigPlot++ program. ${ }^{42}$

\section{Results and Discussion}

\section{Molecular Docking Simulation}

Molecular docking simulations are normally employed to probe the binding affinity between ligands and proteins. Autodock Vina ${ }^{28}$, an open-source docking protocol, is widely used for this purpose. The binding affinity between the ligands and the SARS-CoV-2 Mpro was efficiently estimated using Autodock Vina. ${ }^{28}$ The calculated binding affinity (Table 1 ) is consistent with the respect to experimental binding affinity with an estimated correlation coefficient of $R_{\text {Dock }}=$ $0.72 \pm 0.14$ (cf. 
Table 1 and Figure 2). ${ }^{11-14}$ The obtained values are in good agreement with the docking results of these ligands to SARS-CoV-2 Mpro dimer (PDB ID 6XBG) ${ }^{43}$ with a value of $R_{\text {Dock }}^{\text {Dimer }}=0.74$ (Figure S1 of the Supplementary (ESI) file). Details are shown in Table S1 and Figure S1 of the ESI file. The consistency reveals that the monomer SARS-CoV-2 Mpro can be used as a target for computer-aided drug design aiming prevent SARS-CoV-2 Mpro. Moreover, the root-meansquare error (RMSE) with respect to experiment was estimated as $R M S E=2.42 \pm 0.22 \mathrm{kcal} \mathrm{mol}^{-1}$ (Figure 2). ${ }^{11-14}$ It should be noted that the obtained results are consistent with the recent work which reported the corresponding values of $R_{\text {Dock }}=0.82 \pm 0.08$ and $R M S E=2.28 \pm 0.21 \mathrm{kcal} \mathrm{mol}^{-1} .44$ 
Table 1. The obtained values of the docking simulations.

\begin{tabular}{cccc}
\hline $\mathbf{N}^{\mathbf{0}}$ & Name & $\Delta \mathbf{G}_{\text {Dock }}{ }^{\mathbf{a}}$ & $\Delta \mathbf{G}_{\mathbf{E X P}} \mathbf{b}^{\mathbf{b}}$ \\
$\mathbf{1}$ & $\mathbf{1 1} \mathbf{r}$ & -6.5 & -9.23 \\
2 & $\mathbf{1 3 a}$ & -6.5 & -7.70 \\
3 & $\mathbf{1 3 b}$ & -6.3 & -8.45 \\
4 & $\mathbf{1 1 a}$ & -6.8 & -9.96 \\
5 & $\mathbf{1 1 b}$ & -7.0 & -10.13 \\
6 & Carmofur & -5.7 & -7.86 \\
7 & Disulfiram & -3.8 & -6.89 \\
8 & Ebselen & -5.6 & -8.45 \\
9 & PX-12 & -3.8 & -6.39 \\
10 & Shikonin & -6.1 & -6.58 \\
11 & Tideglusib & -6.6 & -7.95 \\
\hline
\end{tabular}

aThe docking affinity was gained using the Autodock Vina package.

'The experimental binding free energy $\Delta G_{\mathrm{EXP}}$ was roughly computed via the reported IC50 ${ }^{11-14}$ with a supposition that the IC50 value is equal to the inhibition constant $k_{\mathrm{i}}$. The unit is in $\mathrm{kcal} \mathrm{mol}^{-1}$.

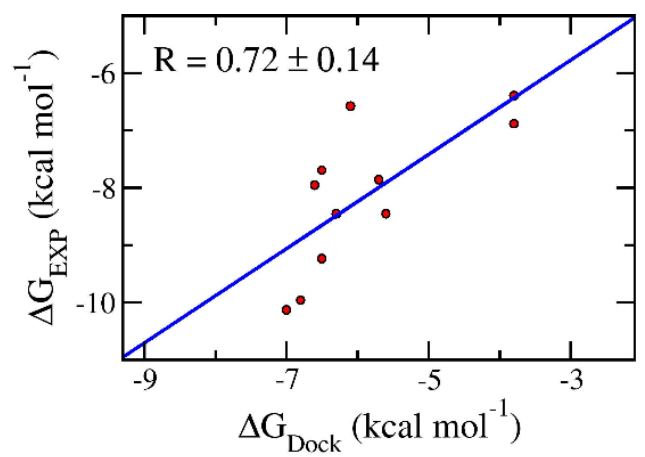

Figure 2. Correlation between molecular docking and experiment. The error of the correlation coefficient was determined via 1000 rounds of the bootstrapping method. ${ }^{41}$

The good docking performance for 11 ligands as shown above gives us the confidence to carry out docking calculations for 36090 compounds in ZINC15 in man only compounds using Autodock Vina package. ${ }^{28}$ However, the compound ZINC000169876613 was skipped because it contains the element silicon for which the docking package has no parameters. The estimated binding free energies for 36089 compounds ranges from -1.8 to $-9.9 \mathrm{kcal} \mathrm{mol}^{-1}$ and have the median of $-5.72 \mathrm{kcal} \mathrm{mol}^{-1}$ and the standard deviation of $1.20 \mathrm{kcal} \mathrm{mol}^{-1}$. We selected one hundred compounds with binding energy to the SARSCoV-2 Mpro lower than $-8.9 \mathrm{kcal} \mathrm{mol}^{-1}$ (Figure 3) for from further investigations using MD simulations. However, thirty-nine of them were discarded from the set since they are just different in protonation states of the same molecules. Overall, sixty-one compounds with two-dimensional interaction diagrams with SARSCoV-2 Mpro (Table S2 of the ESI file) were investigated the unbinding progress via FPL simulations.

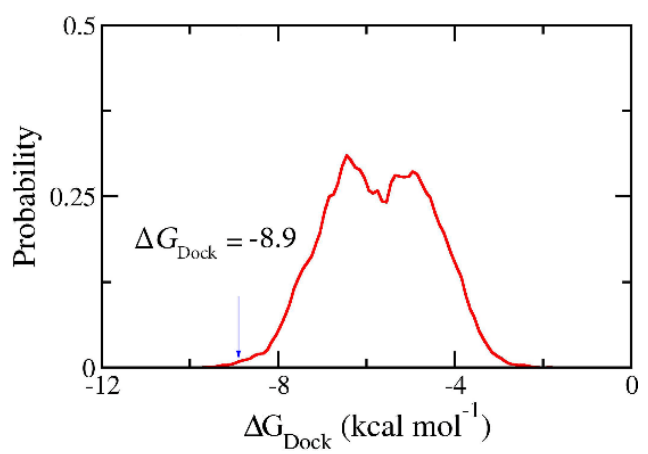

Figure 3. Distribution of the docking energy between 36089 ZINC15 in man only compounds and the SARS-CoV-2 Mpro. The results were gained using Autodock Vina.

\section{Estimating Ligand Affinity using FPL Simulations}

Although, the docking protocol adopts appropriate results compared with experiments (Figure 2), ${ }^{11-13}$ lacking consideration of the receptor dynamics and limiting the number trial position of ligands may cause inaccurate prediction. A more accurate and precise method would be normally employed to refine the docking observation. ${ }^{22,} 45$ Moreover, because the FPL calculation commonly offers accurate and precise results with an reasonable CPU time consumption. ${ }^{46}$ Furthermore, it should be noted that FPL simulations were successfully used in the previous work ${ }^{15}$ to correctly rank the ligand-binding affinity of the $\alpha$-ketoamide $11 r, 13 a$, and $13 b^{11}$ to the SARS-CoV-2 Mpro. The validated calculations were also performed over the additionally available inhibitors including 11a, 11b, carmofur, disulfiram, ebselen, $P X-12$, shikonin, and tideglusib. ${ }^{12-14}$ The obtained results were revealed in Table 2 and Figure S2 of the ESI file. In particular, the mean pulling work $W$ of eleven inhibitors falls in the range from $16.5 \pm 1.7$ to $94.6 \pm 5.0 \mathrm{kcal} \mathrm{mol}^{-1}$, giving a median of 47.2 $\pm 8.6 \mathrm{kcal} \mathrm{mol}^{-1}$. Besides that, the average of the rupture forces forms in the range from $321.2 \pm 26.5$ to $-884.2 \pm 36.5 \mathrm{pN}$, giving an average value of $530.2 \pm 62.6 \mathrm{pN}$. The calculated metrics are in good agreement with the respective experiments, ${ }^{11-14}$ because the correlation coefficient between the mean pulling work and experimental values is of $R_{\mathrm{W}}=-0.76 \pm 0.10$ (Figure 4). The calculated error was computed through 1000 rounds of the bootstrapping method. ${ }^{41}$ Furthermore, the sign of the correlation coefficient $R_{\mathrm{W}}$ implied that the ligand with a stronger binding affinity requires a larger pulling work to dissociate from the SARS-CoV-2 Mpro. Therefore, from linear regression we could estimate the relation between binding free energy $\Delta G_{\mathrm{FPL}}^{\mathrm{Pre}}$ and the pulling work as

$$
\Delta G_{\mathrm{FPL}}^{\mathrm{Pre}}=-0.056 * W-5.512
$$

The precision of the FPL estimation was evaluated by the RMSE with linear regression, giving $R M S E_{\mathrm{W}}=1.03 \pm 0.14 \mathrm{kcal} \mathrm{mol}^{-1}$. The small value of RMSE imply that the FPL simulations can discriminate ligands revealing similar binding free energies. The error was estimated by the standard deviation of 1000 bootstrap samples. ${ }^{41}$ In addition, the measured values $W$ is highly correlated $(R=0.79)$ with the number of residues forming SC contacts with the respective ligand (cf. Table S1 of the ESI). The observed agreement implied that the van der Waals interaction probably dominates the binding process of a ligand to SARS-CoV-2 Mpro. It is in good agreement with the results obtained by the other methods. ${ }^{26,}{ }^{44}$ Overall, the FPL calculations are effective protocol to appraise the ligand-binding 
affinity of the SARS-CoV-2 Mpro with the suitable accuracy and precision.

Table 2. The obtained values of the FPL calculations.

\begin{tabular}{ccccc}
\hline $\mathbf{N}^{\mathbf{0}}$ & Name & $\boldsymbol{F}_{\mathbf{M a x}}{ }^{\mathbf{a}}$ & $\boldsymbol{W}^{\mathbf{b}}$ & $\Delta \mathbf{G}_{\mathbf{E X P}}{ }^{\mathbf{c}}$ \\
\hline 1 & $\mathbf{1 1 r}$ & $857.5 \pm 38.7^{\mathrm{d}}$ & $94.6 \pm 5.0^{\mathrm{e}}$ & -9.23 \\
2 & $\mathbf{1 3 a}$ & $496.0 \pm 32.5^{\mathrm{d}}$ & $43.3 \pm 3.9^{\mathrm{e}}$ & -7.70 \\
3 & $\mathbf{1 3 b}$ & $884.2 \pm 36.5^{\mathrm{d}}$ & $91.9 \pm 3.6^{\mathrm{e}}$ & -8.45 \\
4 & $\mathbf{1 1 a}$ & $701.3 \pm 54.1$ & $70.7 \pm 5.9$ & -9.96 \\
5 & $\mathbf{1 1 b}$ & $718.7 \pm 46.8$ & $74.3 \pm 4.4$ & -10.13 \\
6 & Carmofur & $421.5 \pm 23.9$ & $32.6 \pm 1.8$ & -7.86 \\
7 & Disulfiram & $371.3 \pm 20.3$ & $24.5 \pm 1.9$ & -6.89 \\
8 & Ebselen & $381.0 \pm 34.0$ & $23.5 \pm 2.5$ & -8.45 \\
9 & PX-12 & $321.3 \pm 26.5$ & $16.5 \pm 1.7$ & -6.39 \\
10 & Shikonin & $327.9 \pm 24.4$ & $21.2 \pm 2.1$ & -6.58 \\
11 & Tideglusib & $351.8 \pm 32.4$ & $26.3 \pm 2.4$ & -7.95 \\
\hline
\end{tabular}

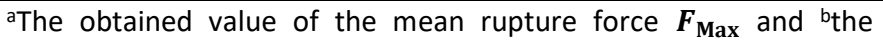
recorded metric of the pulling work $\boldsymbol{W}$. 'The experimental binding free energy $\Delta G_{\mathrm{EXP}}$ was coarsely estimated via the reported IC50 $0^{11-13}$ with a supposition that the IC50 value is equal to the inhibition

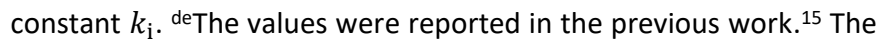
calculated error was the standard error of the average. The unit is in kcal mol-1.

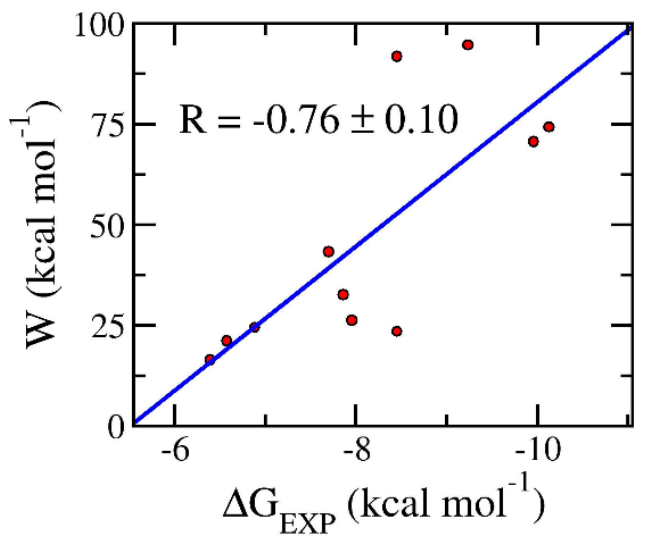

Figure 4. Association between the average of the pulling work $W$ and the binding free energy $\Delta G_{E X P}$ of the respective experiments. Computed values were obtained via the FPL simulations. Experimental metrics were roughly estimated via the reported IC50 $0^{11-13}$ with a hypothesis that the IC50 value is equal to the inhibition constant $k_{i}$ in the recent publications..$^{11-13}$ The linear regression between pulling work and the experiment is $W=$ $-17.993 * \Delta G_{E X P}-98.852$.

The FPL calculations were thus applied to evaluate the binding free energy of docking-top-lead compounds to the SARS-CoV-2 Mpro, which consists of sixty-one compounds listing in Table $\mathbf{3}$ and Table S3 of the ESI file. The mean rupture forces and mean pulling works were found to be diffused in the range from $389.5 \pm 20.9$ to $822.4 \pm$ $40.0 \mathrm{pN}$ and $32.9 \pm 2.6$ to $94.1 \pm 4.7 \mathrm{kcal} \mathrm{mol}^{-1}$, respectively. Particularly, the median of the corresponding metrics are of $574.5 \mathrm{pN}$ and $57.9 \mathrm{kcal} \mathrm{mol}^{-1}$, respectively. Moreover, the predicted binding free energies between ligands and the SARS-CoV-2 Mpro were calculate by using Eq. (1). The value $\Delta G_{\mathrm{FPL}}^{\mathrm{Pre}}$ was thus obtained and shown in Table 3 and Table S3 of the ESI file. It may be argued that a ligand with estimated bining free energy, $\Delta G_{\mathrm{FPL}}^{\mathrm{Pre}}$, less than $-9.00 \mathrm{kcal}$ $\mathrm{mol}^{-1}$ may be able to inhibit the activity of the SARS-CoV-2 Mpro, which would adopt the inhibition constant $k_{i}$ in the sub-micromolar range or smaller. ${ }^{47}$ Therefore, we expect that twenty such compounds to be probable inhibitors for SARS-CoV-2 Mpro activity (Table 3) because of their strong binding affinity. In addition, it may argue that the other compounds, described in in Table S3 of the ESI file, probably adopt less effects on the structure of SARS-CoV-2 Mpro.

Table 3. The obtained values of the docking and FPL simulations.

\begin{tabular}{|c|c|c|c|c|c|c|}
\hline $\mathbf{N}^{0}$ & ZINC ID & Name & $\Delta \mathbf{G}_{\text {Dock }}{ }^{\mathrm{a}}$ & $F_{\text {Max }}{ }^{b}$ & $W^{c}$ & $\Delta G_{\mathrm{FPL}}^{\mathrm{Pre}}$ \\
\hline 1 & ZINC000256110404 & Periandrin V & -9.1 & $782.7 \pm 39.0$ & $94.1 \pm 4.7$ & -10.76 \\
\hline 2 & ZINC000085537131 & Penimocycline & -9.0 & $798.3 \pm 51.2$ & $92.8 \pm 7.0$ & -10.69 \\
\hline 3 & ZINC000100783644 & cis-p-Coumaroylcorosolic acid & -8.9 & $822.4 \pm 40.0$ & $89.5 \pm 4.1$ & -10.51 \\
\hline 4 & ZINC000253527863 & Glycyrrhizin & -9.3 & $598.4 \pm 43.2$ & $86.2 \pm 8.2$ & -10.32 \\
\hline 5 & ZINC000256105139 & Uralsaponin B & -9.7 & $690.6 \pm 33.7$ & $83.6 \pm 3.0$ & -10.17 \\
\hline 6 & ZINC000100783815 & 3-trans-Caffeoyltormentic acid & -8.9 & $731.8 \pm 53.0$ & $77.5 \pm 4.1$ & -9.83 \\
\hline 7 & ZINC000004214527 & Triamcinolone Benetonide & -8.9 & $664.0 \pm 21.6$ & $74.8 \pm 2.3$ & -9.68 \\
\hline 8 & ZINC000028642721 & Sennidin A & -9.5 & $779.7 \pm 58.8$ & $74 \pm 5.2$ & -9.64 \\
\hline 9 & ZINC000100783890 & 23-trans-p-Coumaroyloxytormentic acid & -9.2 & $566.7 \pm 15.1$ & $72.9 \pm 3.4$ & -9.58 \\
\hline 10 & ZINC000098052857 & Evans Blue & -8.9 & $670.2 \pm 56.1$ & $72.7 \pm 6.8$ & -9.56 \\
\hline 11 & ZINC000100783691 & Sanguisorbin $B$ & -8.9 & $616.9 \pm 32.9$ & $71.6 \pm 3.2$ & -9.51 \\
\hline 12 & ZINC000095619992 & Licoricesaponin C2 & -8.9 & $616.3 \pm 44.2$ & $69.9 \pm 7.0$ & -9.41 \\
\hline
\end{tabular}




\begin{tabular}{ccccccc}
13 & ZINC000118937488 & Withangulatin A & -9.0 & $703.5 \pm 31.5$ & $68.5 \pm 3.7$ & -9.33 \\
14 & ZINC000100783660 & trans-3-Feruloylcorosolic acid & -9.2 & $654.9 \pm 23.3$ & $67.3 \pm 2.5$ & -9.26 \\
15 & ZINC000100777487 & Physalin D & -8.9 & $682.3 \pm 32.9$ & $65.8 \pm 2.8$ & -9.18 \\
16 & ZINC000004879678 & Guamecycline & -9.6 & $565.2 \pm 24.8$ & $64.7 \pm 4.8$ & -9.12 \\
17 & ZINC000150354128 & Bis(4-methoxybenzoyl)-3a,29-dihydroxy-8- & -8.9 & $564.8 \pm 39.4$ & $63.8 \pm 3.1$ & -9.07 \\
18 & ZINC000004215464 & multifloren-7-one & -9.2 & $579.3 \pm 38.9$ & $63.5 \pm 4.2$ & -9.05 \\
19 & ZINC000100774273 & Cortisuzol & -8.9 & $696.1 \pm 51.3$ & $62.9 \pm 5.1$ & -9.02 \\
20 & ZINC000073224787 & Rubroskyrin & -9.1 & $573.8 \pm 46.7$ & $62.7 \pm 3.4$ & -9.01 \\
\hline
\end{tabular}

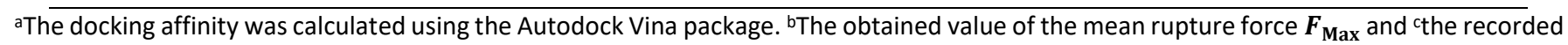
metric of the pulling work $\boldsymbol{W}$. ${ }^{\mathrm{d}}$ The predicted binding free energy $\Delta G_{\mathrm{FPL}}^{\mathrm{Pre}}$ was attained using Eq. (1). The computed error was the standard error of the average. The unit of energy and force are in $\mathrm{kcal} \mathrm{mol}^{-1}$ and $\mathrm{pN}$, respectively.

\section{CPU Time Consumption}

Each SARS-CoV-2 Mpro + ligand complex was simulated over 8 independent FPL simulations, which started from same initial conformation but different random velocity. One FPL trajectory includes $0.1 \mathrm{~ns}$ of NVT, $2.0 \mathrm{~ns}$ of NPT, and $0.5 \mathrm{~ns}$ of SMD simulations. $20.8 \mathrm{~ns}$ of MD simulations was thus performed to appraise the ligandbinding affinity of the SARS-CoV-2 Mpro with 8 various FPL trajectories. It should be noted that one personal computer with AMD Ryzen 9 3950X CPU and RTX 2060 Super acceleration can perform ca. 80 ns of MD simulation per day for the SARS-CoV-2 Mpro + inhibitor system. Therefore, the binding affinity of a ligand to the SARS-CoV-2 Mpro is able to compute 8 times during ca. 6.24 hours. The low CPU requirement permits us to rapidly calculate the binding affinity of many ligands to the SARS-CoV-2 Mpro without any professional computing system.

\section{Conclusions}

We have demonstrated that a combination of molecular docking using Autodock Vina and FPL simulations is able to efficiently estimate the binding affinity of a ligand to the SARS-CoV-2 Mpro. In particular, over eleven available inhibitors for preventing the activity of the SARS-CoV-2 Mpro, Autodock Vina formed a good consistent with the respective experiments. ${ }^{11-13}$ The correlation coefficient and RMSE are measured as $R_{\text {Dock }}=0.72 \pm 0.14$ and $R M S E=2.42 \pm$ $0.22 \mathrm{kcal} \mathrm{mol}^{-1}$, respectively. Moreover, the FPL simulations also adopted results that is in good agreement with these experiments. ${ }^{11-}$ 13 The correlation coefficient and RMSE with linear regression are $R_{\mathrm{W}}=-0.76 \pm 0.10$ and $R M S E_{\mathrm{W}}=1.03 \pm 0.14$ kcal mol-1, respectively.

The combination of two approaches is thus employed to predict probable inhibitors for the SARS-CoV-2 Mpro. A shortlist consisting of sixty-one compounds was found after 36089 compounds were docked to the binding pocket of the SARS-CoV-2 Mpro. The obtained results were then refined by utilizing the FPL calculations. Twenty compounds were finally suggested that they are able to prevent the activity of the SARS-CoV-2 Mpro because they have low $\Delta G_{\mathrm{FPL}}^{\mathrm{Pre}}$, which is smaller than $-9.00 \mathrm{kcal} \mathrm{mol}^{-1}$. Further investigation using in vitro and/or in vivo studies should be carried out to validate the obtained results.

In addition, as discussed above, the requirement of CPU time is quite low. The combination of the Autodock Vina and FPL simulations are efficient way to rapid screening a large number of trial ligand for the SARS-CoV-2 Mpro. Especially, the computations can be carried out at home using an affordable PC with AMD CPU and Nvidia RTX GPU card acceleration.

\section{Acknowledgements}

This work was supported by Vietnam National Foundation for Science \& Technology Development (NAFOSTED) grant \#104.992019.57.

\section{References}

1. C. L. Huang, Y. M. Wang, X. W. Li, L. L. Ren, J. P. Zhao, Y. Hu, L. Zhang, G. H. Fan, J. Y. Xu, X. Y. Gu, Z. S. Cheng, T. Yu, J. A. Xia, Y. Wei, W. J. Wu, X. L. Xie, W. Yin, H. Li, M. Liu, Y. Xiao, H. Gao, L. Guo, J. G. Xie, G. F. Wang, R. M. Jiang, Z. C. Gao, Q. Jin, J. W. Wang and B. Cao, Lancet, 2020, 395, 497-506.

2. C. Wang, P. W. Horby, F. G. Hayden and G. F. Gao, Lancet, 2020, 395, 470-473.

3. C. Yu Wai, Y. Chin-Pang and W. Kwok-Yin, F1000Res, 2020, 9, 129.

4. J. F. W. Chan, S. F. Yuan, K. H. Kok, K. K. W. To, H. Chu, J. Yang, F. F. Xing, J. L. Liu, C. C. Y. Yip, R. W. S. Poon, H. W. Tsoi, S. K. F. Lo, K. H. Chan, V. K. M. Poon, W. M. Chan, J. D. Ip, J. P. Cai, V. C. C. Cheng, H. L. Chen, C. K. M. Hui and K. Y. Yuen, Lancet, 2020, 395, 514-523.

5. N. van Doremalen, T. Bushmaker, D. H. Morris, M. G. Holbrook, A. Gamble, B. N. Williamson, A. Tamin, J. L. Harcourt, N. J. Thornburg, S. I. Gerber, J. O. Lloyd-Smith, E. de Wit and V. J. Munster, N Engl J Med, 2020, DOI: 10.1056/NEJMc2004973.

6. WHO, Coronavirus disease 2019 (COVID-19) Situation Report - 52).

7. worldometrics, COVID-19 Coronavirus Pandemic, https://www.worldometers.info/coronavirus/).

8. P. Zhou, X.-L. Yang, X.-G. Wang, B. Hu, L. Zhang, W. Zhang, H.-R. Si, Y. Zhu, B. Li, C.-L. Huang, H.-D. Chen, J. Chen, Y. Luo, H. Guo, R.-D. Jiang, M.-Q. Liu, Y. Chen, X.-R. Shen, X. Wang, X.-S. Zheng, K. Zhao, Q.-J. Chen, F. Deng, L.-L. Liu, B. Yan, F.-X. Zhan, Y.-Y. Wang, G.-F. Xiao and Z.-L. Shi, Nature, 2020, 579, 270-273.

9. D. Schoeman and B. C. Fielding, Virology, 2019, 16, 69. 10. C. M. Fauquet and D. Fargette, Virology, 2005, 2, 64. 

S. Becker, K. Rox and R. Hilgenfeld, Science, 2020, 368, 409412.

12. Z. Jin, Y. Zhao, Y. Sun, B. Zhang, H. Wang, Y. Wu, Y. Zhu, C. Zhu, T. Hu, X. Du, Y. Duan, J. Yu, X. Yang, X. Yang, K. Yang, X. Liu, L. W. Guddat, G. Xiao, L. Zhang, H. Yang and Z. Rao, Nat Struct Mol Biol, 2020, 27, 529-532.

13. W. Dai, B. Zhang, H. Su, J. Li, Y. Zhao, X. Xie, Z. Jin, F. Liu, C. Li, Y. Li, F. Bai, H. Wang, X. Cheng, X. Cen, S. Hu, X. Yang, J. Wang, X. Liu, G. Xiao, H. Jiang, Z. Rao, L.-K. Zhang, Y. Xu, H. Yang and H. Liu, Science, 2020, DOI: 10.1126/science.abb4489, eabb4489.

14. Z. Jin, X. Du, Y. Xu, Y. Deng, M. Liu, Y. Zhao, B. Zhang, X. Li, L. Zhang, C. Peng, Y. Duan, J. Yu, L. Wang, K. Yang, F. Liu, R. Jiang, X. Yang, T. You, X. Liu, X. Yang, F. Bai, H. Liu, X. Liu, L. W. Guddat, W. Xu, G. Xiao, C. Qin, Z. Shi, H. Jiang, Z. Rao and H. Yang, Nature, 2020, 582, 289-293.

15. S. T. Ngo, N. Quynh Anh Pham, L. Thi Le, D.-H. Pham and V. V. Vu, J. Chem. Inf. Model., 2020, DOI: 10.1021/acs.jcim.0c00491.

16. A.-S. Abd Al-Aziz A., A. Ibrahim, Y. Arpita and P. Raymond A., Computational Design of Potent Inhibitors for SARSCoV-2's Main Protease, 2020.

17. K. Muhammet, Pharmacophore Analyses of SARS-CoV-2 Active Main Protease Inhibitors Using Pharmacophore Query and Docking Study, 2020.

18. K. Gao, D. D. Nguyen, J. Chen, R. Wang and G.-W. Wei, J. Phys. Chem. Lett, 2020, DOI: 10.1021/acs.jpclett.0c01579, 5373-5382.

19. G. R. Marshall, Ann. Rev. Pharmacol. Toxicol., 1987, 27, 193-213.

20. N. Homeyer, F. Stoll, A. Hillisch and H. Gohlke, J. Chem Theory Comput., 2014, 10, 3331-3344.

21. N. T. Mai, N. T. Lan, T. Y. Vu, P. T. Mai Duong, N. T. Tung and H. T. Thu Phung, J. Mol. Graph. Modell., 2020, DOI: https://doi.org/10.1016/i.jmgm.2020.107648, 107648.

$22 . \quad$ P.-T. Tran, V.-H. Hoang, J. Lee, T. T. T. Hien, N. T. Tung and S. T. Ngo, RSC Adv, 2019, 9, 29619-29627.

23. W. Yu and A. D. MacKerell, in Antibiotics: Methods and Protocols, ed. P. Sass, Springer New York, New York, NY, 2017, DOI: 10.1007/978-1-4939-6634-9 5, pp. 85-106.

24. T. Sterling and J. J. Irwin, J. Chem. Inf. Model., 2015, 55, 2324-2337.

25. R. Hatada, K. Okuwaki, Y. Mochizuki, Y. Handa, K. Fukuzawa, Y. Komeiji, Y. Okiyama and S. Tanaka, J. Chem. Inf. Model., 2020, 60, 3593-3602.

26. J. Wang, J. Chem. Inf. Model., 2020, 60, 3277-3286.

27. S. Kim, P. A. Thiessen, E. E. Bolton, J. Chen, G. Fu, A. Gindulyte, L. Han, J. He, S. He, B. A. Shoemaker, J. Wang, B. Yu, J. Zhang and S. H. Bryant, Nucleic Acids Res., 2016, 44, D1202-D1213.

$28 . \quad$ O. Trott and A. J. Olson, J. Comput. Chem., 2010, 31, 455461.

29. N. T. Nguyen, T. H. Nguyen, T. N. H. Pham, N. T. Huy, M. V. Bay, M. Q. Pham, P. C. Nam, V. V. Vu and S. T. Ngo, J. Chem. Inf.Model., 2020, 60, 204-211.

30. M. J. Abraham, T. Murtola, R. Schulz, S. Páll, J. C. Smith, B. Hess and E. Lindahl, SoftwareX, 2015, 1-2, 19-25.

31. A. E. Aliev, M. Kulke, H. S. Khaneja, V. Chudasama, T. D. Sheppard and R. M. Lanigan, Proteins: Struct., Funct., Bioinf., 2014, 82, 195-215.

32. W. L. Jorgensen, J. Chandrasekhar, J. D. Madura, R. W. Impey and M. L. Klein, J. Chem. Phys., 1983, 79, 926-935.
33. J. Wang, W. Wang, P. A. Kollman and D. A. Case, J Mol Graph Model, 2006, 25, 247-260.

34. D. A. Case, I. Y. Ben-Shalom, S. R. Brozell, D. S. Cerutti, T. E. C. Cheatham, III, V.W.D. , T. A. Darden, R. E. Duke, D. Ghoreishi, M. K. Gilson, H. Gohlke, A. W. Goetz, D. Greene, R. Harris, N. Homeyer, Y. Huang, S. Izadi, A. Kovalenko, T. Kurtzman, T. S. Lee, S. LeGrand, P. Li, C. Lin, J. Liu, T. Luchko, R. Luo, D. J. Mermelstein, K. M. Merz, Y. Miao, G. Monard, C. Nguyen, H. Nguyen, I. Omelyan, A. Onufriev, F. Pan, R. Qi, D. R. Roe, A. Roitberg, C. Sagui, S. Schott-Verdugo, J. Shen, C. L. Simmerling, J. Smith, R. SalomonFerrer, J. Swails, R. C. Walker, J. Wang, H. Wei, R. M. Wolf, X. Wu, L. Xiao, Y. D.M. and a. K. P.A., University of California, San Francisco, 2018.

35. A. W. Sousa da Silva and W. F. Vranken, BMC Research Notes, 2012, 5, 1-8.

36. J. Wang, R. M. Wolf, J. W. Caldwell, P. A. Kollman and D. A. Case, J. Comput. Chem., 2004, 25, 1157-1174.

37. H. Zhang, C. Yin, Y. Jiang and D. van der Spoel, J. Chem. Inf. Model., 2018, 58, 1037-1052.

38. H. Zhang, Y. Jiang, Z. Cui and C. Yin, J. Chem. Inf. Model., 2018, 58, 1669-1681.

39. S. T. Ngo, H. M. Hung and M. T. Nguyen, J. Comput. Chem., 2016, 37, 2734-2742.

$40 . \quad$ S. T. Ngo, M. T. Nguyen and M. T. Nguyen, Chem. Phys. Lett., 2017, 676, 12-17.

B. Efron, Ann. Stat., 1979, 7, 1-26.

41.

42.

R. A. Laskowski and M. B. Swindells, J. Chem. Inf. Model., 2011, 51, 2778-2786

43. C. Ma, M. Sacco, Y. Chen and J. Wang, To be published. 2020.

44. N. Son Tung, N. Hung Minh, H. Le Thi Thuy, Q. Pham Minh, T. Vi Khanh, T. Nguyen Thanh and V. Van, Assessing Potential Inhibitors for SARS-CoV-2 Main Protease from Available Drugs using Free Energy Perturbation Simulations, 2020.

$45 . \quad$ S. T. Ngo, S.-T. Fang, S.-H. Huang, C.-L. Chou, P. D. Q. Huy, M. S. Li and Y.-C. Chen, J. Chem. Inf. Model., 2016, 56, 13441356.

46. N. M. Tam, K. B. Vu, V. V. Vu and S. T. Ngo, Chem. Phys. Lett., 2018, 701, 65-71.

47. G. Subramanian, B. Ramsundar, V. Pande and R. A. Denny, J. Chem. Inf. Model., 2016, 56, 1936-1949. 